gen oxidation there, or that oxidation is suppressed at the membrane-covered inner electrode. Whether other physical phenomena, uniquely associated with the small size of the device, can affect the transport of reactants and cause spontaneous polarization of the cell remains to be investigated. The answer may lead to devices with improved performance.

The thin-film fuel cell is only one of several recently described microscopic electrochemical devices of various capabilities that can be fabricated by lithographic techniques. Extremely small dimensions allow the use of relatively poor electrical conductors (pseudoboehmite in the case of Dyer's fuel cell) as components without excessive ohmic losses. The use of microlithography also opens up the possibility of integrating electrochemistry with electronics; in the resulting composite systems an electrochemical cell interfaces an electronic device with the chemical 'real world', receiving or transmitting chemical signals. For sensor applications, an additional multiplex advantage is gained, because components that are selective for different chemical signals can be applied to an array of transistor gates or photodiodes to be read simultaneously.

In an interesting variation on this theme, Parce et al. ${ }^{3}$ have shown that a silicon chip can be fabricated into a 'microphysiometer' by etching an array of $50-\mu \mathrm{m}$ square wells in the silicon and covering them with thin insulating layers of $\mathrm{SiO}_{2}$ and $\mathrm{Si}_{3} \mathrm{~N}_{4}$. A glass cover slip placed over the array traps a small number of living cells within each well. Because living cells produce $\mathrm{CO}_{2}$ and lactate, the change of $\mathrm{pH}$ within the wells is related to their metabolic rate. The $\mathrm{Si}_{3} \mathrm{~N}_{4}$ overlayer is $\mathrm{pH}$ sensitive, and the device therefore acts as a microscopic $\mathrm{pH}$ meter, reading the transient metabolic response of as few as a thousand cells to various chemical and biochemical stimuli. It is likely to be useful for in vitro screening of therapeutic drugs, and detection of biochemicals, viruses, and products released by one cell that affect a second cell.

Aviram and co-workers ${ }^{4.5}$ have published experimental and theoretical papers on what may be the ultimate goal in integrated electrochemical $/ \mathrm{microelec}$ tronic devices - single electroactive molecules that act as switching elements in electronic circuits. Molecular-orbital calculations indicate that molecules consisting of orthogonal conducting polymer strands, linked by a spiroalkane bridge, could form the basis of logic gates. An electric field of the order of $10^{6} \mathrm{~V} \mathrm{~cm}-1$ applied parallel to the bridge would cause an electron to be transferred between strands, causing them to switch between conductive and nonconductive states ${ }^{5}$.

In related experiments, a catechol/ orthoquinone molecule was prepared as a self-assembling monolayer on a gold surface, and interrogated with the tip of a scanning tunnelling microscope (STM) ${ }^{4}$. The molecule is orientated in such a way that the catechol moiety is bound to the gold, through a thioether group, and the orthoquinone group is nearer the STM tip. When the gold is positive with respect to the STM tip, a direct current flows, presumably because the catechol is oxidized and the quinone is reduced; the resulting semiquinone-semiquinone diradical is thought to be a good electrical conductor. In line with this interpretation, no current flows when the polarity is reversed. Similar diode-like behaviour has been observed using an STM tip to address individual atomic defects on the surface of silicon ${ }^{6,7}$.

The wiring and interconnection of individual molecules is obviously the greatest challenge in this work, and chemical rules which may be useful are now beginning to emerge. Laibinis et al. ${ }^{8}$ have shown, for example, that 'hard' and 'soft' acid-base interactions between chemical functional groups and metal or metal-oxide surfaces can be used to direct molecules to bind in specific regions of a microelectronic array. With an interdigitated array of oxidized aluminium and gold microwires, they showed that alkanethiols and alkylcarboxylic acids dissolved in the same solution bind exclusively the gold and aluminium surfaces, respectively. One can easily envisage molecules with thiol and carboxylate functionalities at either end which could bridge between dissimilar metal contacts in a microelectronic structure. True molecular electronics based on such structures would require a practical technique (nonexistent at present) for arranging patterns of these wires on the scale of molecular dimensions.

Although Dyer's thin-film fuel cell produces small currents, it is possible that an improved understanding of its operation will lead to successful high-power applications, as in electric vehicle propulsion. The fuel cell is at present interesting as a power source for low-current applications, for example in remote sensing and microelectronics. One can now imagine integrated microelectrochemical devices, based on this emerging technology, that incorporate the elements of energy conversion, chemical sensing and molecular computing.

Thomas E. Mallouk is in the Department of Chemistry and Biochemistry, University of Texas at Austin, Austin, Texas 78712, USA.

1. Dyer, C.K. Nature $343,547-548$ (1990)

2. Kordesch, K. \& Oliveira, J.C.T. Int. J. Hydrogen Energy 13 411-427 (1988)

411-427 (1988).
3. Parce, J.W. et al. Science 246, 243-247 (1989).

4. Aviram, A., Joachim, C. \& Pomerantz, M. Chem. Phys. Lett 146, $490-495$ (1988)

5. Aviram, A. J. Am. chem. Soc. 110, 5687-5692 (1988)

6. Bedrossian, P., Chen, D.M., Mortensen, K. \& Golovchenko, J.A. Nature 342, 258-260 (1989) 7. Lyo, I.-W \& Avouris, P. Science 245, 1369-1371 (1989).
8. Laibinis, P.E., Hickman, J.J., Wrighton, M.S. \& Whitesides, G.M. Science 245, 845-847 (1989).

\section{Light engineering}

A RADIOTHERAPIST treating a cancer will try to maximize the radiation dose to the cancer while minimizing it for the surrounding tissue. This can be achieved by aiming the beam successively from many directions, intersecting at the cancer. Daedalus is now generalizing this strategy. He claims that a complete set of beams, each of a properly computed intensity and direction, could impose any desired pattern of radiation intensity on the interior of the object at which they were aimed.

The process is simply the converse of $\mathrm{X}$-ray tomography. An X-ray beam projected through an object emerges with an intensity that sums the $\mathrm{X}$-ray absorption densities along its path. A tomographic scanner records the emergent beam intensity for every possible line of intersection with the object, so as to compute the density at each point inside it. Daedalus's inverse tomograph projects a pre-computed beam intensity along every possible line of intersection with its target, so as to deliver a specific summed radiation dose to each point inside it.

While the inverse-tomograph may well sharpen radiotherapy, Daedalus wants to apply it to engineering. Aimed under computer control at a tank of radiationsensitive liquid monomer, its X-ray or ultraviolet beam could 'sculpt' a chosen shape in radiation density. The monomer would photopolymerize into a perfect copy of that shape, which could then be pulled out of the tank. Shapes impossible to mould, with arbitrarily complex reentrants, holes, or internal detail, could be created cheaply and flexibly by pure software, and could be updated and modified at will.

More subtly still, the inverse tomograph could make objects whose physical properties varied from point to point. A graded radiation pattern could lightly crosslink the polymer to a pliant rubber at one point, while setting it firmly to rigid polymer at another. Crash helmets with a hard skin but soft interior, luggage with a rigid frame but yielding exterior, shock-proof mountings with a complex pattern of solid, elastic and damping regions, could all be formed in one operation from a single material.

Daedalus is even designing a combined inverse tomograph and bubble chamber, to produce variable density foamed plastics. A volatile liquid monomer under reduced pressure might boil and foam in highly irradiated regions. Plastic components could thus be made hard and dense at the surface or in regions subject to heavy loads, but foam-light elsewhere. The engineering dream of a complete structure free of joints and fastenings, as light as possible and just strong enough at each point to bear the local load, would be a reality at last.

David Jones 